Canadian Oncology

Nursing Journal

Revue canadienne

de soins infirmiers

en oncologie

Volume 28, Issue 3 • Summer 2018

elSSN: 2368-8076 


\title{
Médiateurs potentiels d'amélioration de la neuropathie périphérique chimio- induite douloureuse par une intervention cognitivocomportementale en ligne
}

\author{
par Robert Knoerl, Debra L. Barton, Janean E. Holden, John C. Krauss, Beth LaVasseur et Ellen M.L. Smith
}

RÉSUMÉ

Objet : Des données préliminaires ont révélé qu'une intervention autoguidée cognitivocomportementale de gestion de la douleur (PROSPECT) était efficace contre la neuropathie périphérique chronique douloureuse induite par chimiothérapie (NPCI), mais le mécanisme d'action demeure inconnu. L'objectif de cette analyse secondaire a consisté à déterminer si les changements par rapport à l'anxiété, à la dépression, aux troubles du sommeil ou à la fatigue diminuaient la douleur après l'utilisation de PROSPECT chez les patients atteints de NPCI.

Méthodologie : En tout, 60 participants ont été sélectionnés au hasard pour recevoir soit l'intervention autoguidée cognitivocomportementale contre la douleur (accès pendant huit semaines), soit le traitement habituel. Un journal sur sept jours de suivi de la douleur due à la NPCI et le système PROMIS (Patient Reported Outcomes Measurement Information System) de mesure de l'anxiété, de la dépression, de la fatigue et des troubles du sommeil

\section{AU SUJET DES AUTEURS}

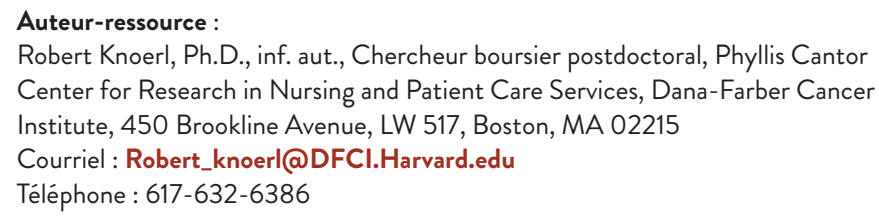

Robert Knoerl, Ph.D., inf. aut., Chercheur boursier postdoctoral, Phyllis Cantor Center for Research in Nursing and Patient Care Services, Dana-Farber Cancer Institute, 450 Brookline Avenue, LW 517, Boston, MA 02215

Courriel : Robert_knoerl@DFCl.Harvard.edu

Téléphone : 617-632-6386

Debra L. Barton, inf. aut., Ph.D., AOCN, FAAN, Mary Lou Willard, professeure française de soins infirmiers; University of Michigan Medical School, Ann Arbor, M.I.; courriel : debbartn@umich.edu

Janean E. Holden, Ph.D., inf. aut., FAAN, Barbara A. Therrien, professeure en soins infirmiers, University of Michigan School of Nursing, Ann Arbor, M.I.; courriel : holdenje@umich.edu

John C. Krauss, M.D., Professeure adjointe, University of Michigan Comprehensive Cancer Center, Ann Arbor, M.I.; courriel :

jkrauss@med.umich.edu

Beth LaVasseur, inf. aut., M.Sc., Directrice de la recherche et des opérations, St. Joseph Mercy Hospital, Ann Arbor, M.I.; courriel :

beth.lavasseur@st.joeshealth.org

Ellen M.L. Smith, Ph.D., APRN, AOCN®, FAAN, Professeurs adjointe, University of Michigan School of Nursing, Ann Arbor, M.l.; courriel : ellenls@umich.edu

DOI:10.5737/23688076283184190 ont été utilisés avant et après l'étude (huit semaines). L'analyse de la médiation causale a été utilisée pour quantifier les médiateurs d'amélioration quant aux douleurs les plus intenses.

Résultats : Aucun des médiateurs hypothétiques n'a eu un effet statistiquement important sur les douleurs les plus fortes $(n=38)$.

Implications : D’autres recherches sont nécessaires pour déterminer les médiateurs potentiels d'intensité de la douleur qui peuvent être ciblés par des stratégies cognitivocomportementales spécifiques afin d'améliorer la gravité de la douleur de la CIPN.

Mots clés : Douleur chronique, neuropathie périphérique chimio-induite, intervention cognitivocomportementale, maladie du système nerveux périphérique/chimio-induite

\section{INTRODUCTION}

T a douleur chronique causée par la neuropathie ـpériphérique chimio-induite survient chez $40 \%$ des personnes recevant des agents chimiothérapeutiques neurotoxiques comme les platines ou les taxanes (Kautio, Haanpää, Kautiainen, Kalso et Saarto, 2011; Smith, Cohen, Pett et Beck, 2011). La douleur chronique de la NPCI se caractérise par des sensations de brûlures, d'engourdissements et de picotements dans les mains et les pieds qui peuvent persister des mois ou des années après la fin de la chimiothérapie neurotoxique et peuvent avoir une incidence négative sur la qualité de vie et la fonction physique (Beijers, Mols, Dercksen, Driessen et Vreugdenhil, 2014; Smith et al., 2014).

Actuellement, il n'existe qu'un seul traitement pharmacologique (duloxétine $60 \mathrm{mg} /$ jour) et aucun traitement non pharmacologique n'est recommandé pour la prise en charge de la douleur chronique de la NPCI (Hershman et al., 2014; Smith et al., 2013). Un essai randomisé et contrôlé par liste d'attente (Knoerl, Smith et al., 2017) a examiné l'efficacité d'une intervention autoguidée en ligne sur la gestion de la douleur cognitivocomportementale, appelée Proactive Self-management Program for Effects of Cancer Treatment (PROSPECT) sur l'intensité des douleurs causées par la NPCI. Des résultats ont démontré que l'utilisation de PROSPECT réduisait de façon significative l'intensité de la douleur par rapport aux personnes recevant des soins habituels $(p=0,046)$. Malgré ces résultats statistiquement significatifs, seulement $47 \%$ des personnes recevant PROSPECT ont connu une réduction de la douleur d'au moins $10 \%$. PROSPECT n'a pas fonctionné chez tous les participants, et on sait peu de choses sur la façon dont la gestion de la douleur cognitivocomportementale fonctionne 
pour améliorer la douleur due à la NPCI. Par conséquent, des recherches supplémentaires sont nécessaires pour déterminer les médiateurs de l'intensité de la douleur qui peuvent être ciblés par PROSPECT afin d'améliorer l'effet de l'intervention sur la sévérité de la douleur due à la NPCI.

Un certain nombre d'études prouvent l'existence de relations entre plusieurs variables médiatrices (c.-à-d. l'anxiété, la dépression, la fatigue et les troubles du sommeil) et l'intensité douloureuse chronique de la NPCI (Hershman et al., 2016; Smith et al., 2015). Ces relations sont en outre soutenues par des mécanismes pathophysiologiques sous-jacents communs : 1) changements neurobiologiques (Boakye et al., 2016; Nudelman et al., 2016; Zhuo, 2016); 2) réponse au stress prononcée (p. ex. perturbation de l'axe hypothalomo-hyphyso-surrénalien); 3) dysrégulation de la cytokine; 4) dysrégulation de la 5-HT et de la noradrénaline (Barsevick, Frost, Zwinderman, Hall et Halyard, 2010; Boakye et al., 2016). La gestion cognitivocomportementale de la douleur peut contribuer à diminuer l'intensité de la douleur par l'induction de changements structurels dans le cerveau, ce qui peut par la suite influencer les voies nociceptives inhibitrices descendantes par la libération de norépinéphrine et de sérotonine (Jensen et al., 2012; Seminowicz et al., 2013). Ainsi, parce que la douleur chronique de la NPCI coexiste avec des symptômes qui partagent des mécanismes pathophysiologiques similaires et que la gestion de la douleur cognitivocomportementale cible des mécanismes communs à tous les symptômes, il est possible que l'amélioration de l'anxiété, de la dépression, de la fatigue ou des troubles du sommeil puisse également améliorer la douleur. Cependant, nous n'avons pas connaissance d'études publiées qui ont examiné les médiateurs d'amélioration de l'intensité de la douleur chronique douloureuse de la NPCI suite à une prise en charge de la douleur cognitivocomportementale autoguidée. Le but de cette analyse secondaire était d'explorer l'effet médiateur des changements moyens dans les troubles du sommeil, l'anxiété, la dépression et la fatigue sur l'intensité de la douleur la plus forte après PROSPECT chez les personnes atteintes de douleurs chroniques de la NPCI.

\section{MATÉRIEL ET MÉTHODOLOGIE}

\section{Devis, contexte et échantillon}

Cette étude est une analyse secondaire d'un essai pilote randomisé et contrôlé par liste d'attente testant l'effet de PROSPECT sur l'intensité de la plus forte douleur liée à la NPCI en comparaison avec les patients recevant le traitement habituel (Knoerl et al., 2017). En bref, soixante patients souffrant de douleur chronique due à la NPCI (intensité des douleurs les plus intenses dues à la NPCI d'au moins $4 / 10 \geq$ trois mois depuis la fin de la chimiothérapie neurotoxique) ont été recrutés dans cinq centres universitaires ou communautaires de traitement sur une période de six mois. Les patients qui présentaient une neuropathie due à d'autres causes ou qui prévoyaient recevoir une chimiothérapie neurotoxique pendant l'étude ont été exclus. Le protocole de l'étude a été approuvé par le comité d'examen des établissements concernés et les participants ont donné leur consentement éclairé par écrit.

\section{Mesures}

Une échelle d'évaluation numérique de 11 points (de 0 à 10 , « 10 » représentant la pire douleur imaginable) a été utilisée pour mesurer l'intensité de la douleur liée à la NPCI (Cleeland et Ryan, 1994); elle a été administrée par l'entremise d'un journal de suivi sur sept jours au début de l'étude et après huit semaines. La moyenne des réponses des participants dans le journal sur sept jours a été calculée à chacun de ces deux points temporels. Diverses mesures du système PROMIS (Patient Reported Outcome Measurement Information System) ont en outre été utilisées pour quantifier l'anxiété, la dépression, la fatigue et les troubles du sommeil dans l'échantillon recruté (Cella et al., 2007). L'outil PROMIS d'évaluation de l'anxiété 4a (4 items; 1 = jamais; 5 = toujours; échelle de notation totale transformée 40,3 - 81,6) mesure la peur, l'inquiétude et l'inconfort déclarés au cours des sept derniers jours (Kroenke, Yu, Wu, Kean et Monahan, 2014). L'outil PROMIS d'évaluation de la dépression et la détresse émotionnelle 4a (4 items; 1 = jamais; 5 = toujours; échelle de score total transformé 41,0 - 79,4) examine la tristesse, la perception de soi, la solitude et l'objectif personnel du patient des sept derniers jours (Bartlett et al., 2015; Kroenke et al., 2014). L'outil PROMIS d'évaluation de la fatigue 4a (4 items; 1 = pas du tout; 5 = beaucoup; score total transformé 33,7 - 75,8) mesure les sentiments de fatigue et d'épuisement qui diminuent possiblement la capacité d'une personne à accomplir ses activités quotidiennes et à fonctionner normalement dans ses rôles familiaux/personnels (Bartlett et al., 2015). L'outil PROMIS d'évaluation des troubles du sommeil 8a (4 items; 1 = pas du tout; 5 = beaucoup; échelle de notation totale transformée 30,0-80,1) mesure les perceptions déclarées par le patient quant à la vigilance, la somnolence, la fatigue pendant la journée et les troubles fonctionnels associés au sommeil au cours des sept derniers jours (Yu et al., 2012).

\section{Procédures}

Au départ, avant la randomisation, les participants ont rempli le premier jour du journal de la douleur NPCI et les sous-échelles PROMIS sur une tablette. Le chercheur principal a ensuite randomisé les participants à un groupe d'étude (huit semaines de PROSPECT ou traitement habituel) dans un rapport de r:I à l'aide d'un tableau de nombres aléatoires généré par ordinateur. Les participants ont ensuite reçu une copie papier du journal de suivi sur sept jours des douleurs les plus intenses causées par la NPCI (pour compléter les six jours restants du journal) et ont soumis leurs scores par le biais d'un lien de sondage envoyé par courriel. Après avoir rempli le journal sur la douleur, les participants ont reçu par courriel le lien vers le site Web PROSPECT, ou de l'information sur le traitement habituel pour le groupe témoin. Le site Web PROSPECT contient des stratégies cognitivocomportementales (p. ex. espacement des activités pour gérer la fatigue, relaxation musculaire progressive/respiration profonde pour l'anxiété/la dépression, stratégies d'hygiène du sommeil pour les troubles du sommeil) et des informations sur l'autogestion (p. ex. communication patient-fournisseur au sujet des symptômes et établissement d'objectifs) conçues pour aider les 
Médiateurs hypothétiques

\begin{tabular}{|c|c|}
\hline Anxiété & $\begin{array}{l}\text { Difficultés } \\
\text { de sommeil }\end{array}$ \\
\hline Dépression & Fatigue \\
\hline
\end{tabular}

A

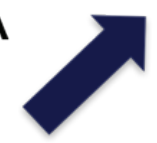

Variable indépendante

PROSPECT

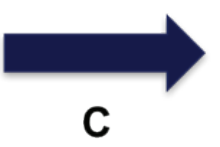

B

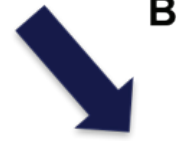

Variable dépendante

Douleur la plus forte due à la NPCI

Figure 1. Modèle de médiation hypothétique de PROSPECT sur l'intensité la douleur due à la NPCI Cette figure décrit les médiateurs hypothétiques de l'effet de PROSPECT sur l'intensité de la gravité de la douleur liée à la NPCI.

Les parcours « $A$ » et «B » représentent l'effet indirect de PROSPECT sur l'intensité de gravité de la douleur liée à la NPCI tel qu'expliqué à l'aide des médiateurs.

Le parcours « $\mathrm{C} »$ représente l'effet direct de PROSPECT sur l'intensité de la gravité de la douleur liée à la NPCI.

personnes à gérer les symptômes liés au traitement du cancer. Le contenu est transmis par écrit et par vidéo. L'intervention PROSPECT recommande également différents modules et stratégies en fonction des symptômes autodéclarés par les participants. Les participants ont été formés par le chercheur principal au départ sur la façon d'accéder au site Web PROSPECT et de l'utiliser et ont été encouragés à utiliser ce site à leur discrétion. Huit semaines après la randomisation, les participants ont reçu par courriel des versions électroniques du journal sur sept jours des douleurs les plus intenses dues à la NPCI et les mesures PROMIS.

\section{Analyse statistique}

Loutil R-3.4.0 a été utilisé pour l'analyse des données (R Development Core Team, 2017). Léchantillon analysé était fondé sur les personnes qui ont rempli toutes les données du sondage pré/post-test dans l'étude primaire. Dans l'étude primaire, 38 personnes ont fourni des données au début et au bout de huit semaines sur les douleurs les plus intenses causées par la NPCI dans l'étude primaire (Knoerl et al., 2017), mais seulement 37 d'entre elles ont fourni des données complètes pour les résultats secondaires explorés dans cette étude ( $<5 \%$ de données manquantes pour cette analyse). Pour être cohérents avec la taille de l'échantillon de l'étude primaire, nous avons utilisé l'imputation moyenne pour traiter les données manquantes pour un participant (Little, Jorgensen, Lang et Moore, 2014). Nous n'avons pas effectué d'analyse de puissance a priori en raison de la nature exploratoire de cette analyse.
La médiation causale (Imai, Keele et Tingley, 2010) a été utilisée pour déterminer si des changements dans l'anxiété, la dépression, la fatigue ou les troubles du sommeil pouvaient avoir modulé les effets de PROSPECT sur l'amélioration de l'intensité de la douleur la plus intense au cours de la période de traitement de huit semaines (figure 1). L'effet de médiation causale est défini comme l'effet indirect du traitement sur la variable dépendante par l'intermédiaire des médiateurs (parcours « $A$ » et « B » de la figure 1) (Imai et al., 2010). L'effet indirect peut être défini comme le changement du résultat (ici, les douleurs les plus intenses) lorsque la valeur du médiateur (p. ex. l'anxiété) passe de la valeur déclarée par le témoin à la valeur déclarée par le traitement.

Des statistiques descriptives (moyenne, écart-type) ont été calculées au départ et à la semaine 8 pour toutes les données continues (douleurs les plus fortes liées à la NPCI, fatigue, anxiété, dépression et troubles du sommeil). Ensuite, pour évaluer l'effet de PROSPECT sur les médiateurs hypothétiques, on a comparé les scores moyens de la huitième semaine pour l'anxiété, la dépression, les troubles du sommeil et la fatigue entre les groupes au moyen d'une analyse de covariance en ajustant les scores de base. Lorsque les résultats montraient que PROSPECT n'avait aucun effet sur un médiateur hypothétique, alors les analyses de médiation ne pouvaient pas être effectuées, car une variable ne peut être un médiateur de résultat que lorsqu'elle est affectée de façon significative par le traitement (Imai et al., 2010). Par la suite, pour les variables qui ont affiché une amélioration statistiquement significative à la suite de l'utilisation de PROSPECT, nous avons modélisé : 1) les 
Tableau 1. Moyenne des scores à la sous-échelle PROMIS au début et à la semaine 8

\begin{tabular}{|c|c|c|c|}
\hline Résultats $(n=38)^{a}$ & Intervention Moyenne (é.-t.) & Contrôle par liste d'attente Moyenne (é.-t.) & Contraste entre les groupes ${ }^{a}$ \\
\hline Semaine 8 & $56,71(5,90)$ & $54,34(5,86)$ & $\begin{array}{l}B=0,22 ; p=0,87 \\
I C=-2,39 ; 2,82\end{array}$ \\
\hline \multicolumn{4}{|l|}{ Fatigue } \\
\hline Semaine 8 & $56,83(8,41)$ & $51,47(7,92)$ & $\begin{array}{l}B=0,22 ; p=0,92 \\
I C=-3,90 ; 4,33\end{array}$ \\
\hline \multicolumn{4}{|l|}{ Dépression ${ }^{b}$} \\
\hline Départ & $52,79(7,03)$ & $47,89(7,12)$ & $\begin{array}{l}B=2,54 ; p=0,16 \\
I C=-1,03 ; 6,11\end{array}$ \\
\hline \multicolumn{4}{|l|}{ Anxiété } \\
\hline Départ & $54,45(7,27)$ & $50,09(7,82)$ & \multirow{2}{*}{$\begin{array}{l}B=1,38 ; p=0,55 \\
I C=-3,23 ; 5,99\end{array}$} \\
\hline Semaine 8 & $52,62(8,59)$ & $48,63(7,41)$ & \\
\hline \multicolumn{4}{|c|}{$\begin{array}{l}\text { Ce tableau illustre les différences de scores moyens à la huitième semaine pour les variables des difficultés de sommeil, de la fatigue, de } \\
\text { la dépression et de l'anxiété entre les participants utilisant PROSPECT et ceux recevant le traitement habituel (groupe témoin - liste } \\
\text { d'attente). } \\
\text { a Différence dans les scores moyens de la semaine } 8 \text { en ajustant pour les scores de base. } \\
\text { b Les personnes recevant les soins habituels ont connu de plus grandes améliorations. }\end{array}$} \\
\hline
\end{tabular}

médiateurs (scores sur l'anxiété, la dépression, la fatigue et/ou les troubles du sommeil de la semaine 8, respectivement) en fonction du traitement et des covariables de base (c'est-à-dire les scores de base des douleurs les plus intenses); 2) le résultat (score de la semaine 8 de la plus grande intensité de la douleur) en fonction du traitement, du médiateur et des covariables de base (Imai et al., 2010; Imai, Keele, Tingley et Yamamoto, 2015). Ces deux modèles ont ensuite été combinés dans la fonction médiate de la trousse Mediation (Tingley et al., 2014) pour estimer l'effet causal de la médiation et l'IC à $95 \%$ de l'effet causal de la médiation pour chaque modèle de médiation.

\section{RÉSULTATS}

\section{Caractéristiques de l'échantillon}

Les caractéristiques démographiques et les caractéristiques liées au traitement du cancer de l'échantillon recruté ont été décrites précédemment (Knoerl, Smith et al., 2017). Lâge moyen des participants à l'étude était de 61,15 ans $(E T=9,06$ ans, écart $=40-78)$. Léchantillon était principalement féminin (75\%), caucasien $(91,7 \%)$, de niveau collégial $(82,1 \%)$, retraité $(43,3 \%)$, marié $(70 \%)$, et utilisait régulièrement un ordinateur $(85 \%)$. La plupart des participants avaient un cancer du sein (38,3\%) ou gastro-intestinal (43,3\%) et la gravité du cancer variait. Les personnes utilisant PROSPECT présentaient davantage de fatigue et de troubles du sommeil au début de l'étude que les personnes recevant le traitement habituel. De plus, les personnes qui n'ont pas terminé l'étude présentaient un cancer plus avancé (46\% avaient un cancer de stade IV comparativement à $19 \%$ pour les personnes ayant terminé l'étude), mais il n’y avait pas de différences considérables dans la douleur au début ou la gravité des symptômes concomitants entre les personnes ayant terminé l'étude ou non.

\section{Analyse de la médiation}

Le tableau 1 présente les scores moyens pour les variables de l'anxiété, de la dépression, de la fatigue et des troubles liés au sommeil au début de l'étude et à la semaine 8. Il n'y avait pas de différences significatives dans l'anxiété, la fatigue, les troubles du sommeil ou la gravité de la dépression entre les groupes à la huitième semaine $(\mathrm{p}>0,05 ; \mathrm{n}=38)$. Les tendances de l'anxiété, de la dépression et de la fatigue au fil du temps ont également indiqué que PROSPECT n'offrait aucun avantage clair par rapport aux soins habituels. Les tendances de la gravité des troubles du sommeil suggèrent que les personnes recevant PROSPECT présentaient des améliorations constantes au fur et à mesure que l'étude progressait. Néanmoins, comme PROSPECT n'a pas eu d'effet statistiquement significatif sur les médiateurs hypothétiques par rapport au groupe témoin habituel, il n'a pas été possible d'effectuer des analyses de médiation.

\section{DISCUSSION}

Les résultats des analyses de médiation ont révélé qu'aucun des facteurs d'influence hypothétiques sur la douleur chronique liée à la NPCI n'a diminué de façon significative l'intensité de la douleur la plus forte due à la NPCI après l'usage de PROSPECT. Certaines études publiées ont démontré que 
les facteurs émotionnels intervenaient dans l'amélioration de l'intensité de la douleur à la suite de la prise en charge cognitivocomportementale de la douleur, que ce soit en personne (anxiété) ou en ligne (stress et dépression) (DasMahapatra, Chiauzzi, Pujol, Los et Trudeau, 2015; McCracken, Gross et Eccleston, 2002). L'une des raisons pour lesquelles l'anxiété et la dépression n'ont pas été observées comme médiateurs dans la présente étude est que l'intervention PROSPECT ne contenait pas suffisamment de stratégies pour traiter adéquatement ces symptômes. Par exemple, la restructuration cognitive, une stratégie clé de la thérapie cognitivocomportementale contre l'anxiété et la dépression, n'était pas incluse dans PROSPECT (Beck, Beck et S., 2010). Il est prouvé que la restructuration cognitive représente un élément clé des interventions antérieures autoguidées de gestion de la douleur cognitivocomportementale. Des sept essais autoguidés sur la gestion de la douleur cognitivocomportementale examinés par Knoerl, Lavoie Smith et Weisberg (2015), quatre ont eu des effets positifs sur l'anxiété et la dépression. Ces quatre essais se sont particulièrement intéressés à la restructuration cognitive en donnant aux participants l'accès à des modules contenant cette stratégie pendant plusieurs semaines. Inversement, les programmes qui mettaient davantage l'accent sur l'autogestion (p. ex. communication avec le fournisseur de soins, gestion des médicaments, établissement d'objectifs) étaient moins efficaces en ce qui a trait à l'anxiété et à la dépression. Les futurs prototypes de PROSPECT devraient inclure et mettre l'accent sur des modules spécifiques aux stratégies de restructuration cognitive pour cibler les symptômes tels que l'anxiété et la dépression.

On en sait moins sur l'efficacité de la gestion de la douleur cognitivocomportementale pour la fatigue liée à la douleur et les troubles du sommeil chez les personnes qui souffrent de douleur chronique (Knoerl, Lavoie Smith et Weisberg, 2015). Cependant, il existe de nombreuses preuves que la thérapie cognitivocomportementale aide à contrer l'insomnie et la fatigue (Price, Mitchell, Tidy et Hunot, 2008; Zachariae, Lyby, Ritterband et O'Toole, 2016). Par exemple, un récent essai contrôlé randomisé par Ritterband et collègues (2017) a testé une intervention cognitivocomportementale autoguidée pour le sommeil qui incorporait l'hygiène et la restriction du sommeil, le contrôle des stimuli, la prévention des rechutes et des stratégies de restructuration cognitive. Les résultats indiquaient que les patients recevant l'intervention avaient significativement amélioré la gravité de l'insomnie $(\mathrm{p}<0,001)$ par rapport à ceux qui avaient reçu de la formation sur l'insomnie (Ritterband et al., 2017). Les stratégies visant à gérer et à augmenter l'activité physique se sont aussi révélées efficaces contre la fatigue (Larun, Brurberg, Odgaard-Jensen et Price, 2016). Ainsi, les futurs prototypes de PROSPECT pourraient explorer l'ajout de stratégies liées à la restriction du sommeil (p. ex. dormir/se réveiller à certains moments pour reprogrammer la dynamique du sommeil), des stratégies de restructuration cognitive dans le contexte des troubles liés au sommeil, et d'autres façons de gérer et d'accroître l'activité physique pour cibler la fatigue et les troubles du sommeil chez les personnes souffrant de douleurs chroniques liées à la NPCI.
Bien que nous ayons évalué l'effet médiateur de l'anxiété, de la dépression, de la fatigue et des troubles du sommeil sur l'intensité de la douleur de la NPCI la plus forte, il existe d'autres facteurs d'influence que nous n'avons pas mesurés pouvant agir sur l'amélioration de l'intensité de la douleur de la NPCI la plus forte. Plus précisément, les variables cognitives (p. ex. le contrôle perçu de la douleur, le catastrophisme de la douleur et l'auto-efficacité pour gérer la douleur) se sont révélées médiatrices de l'amélioration de la douleur chronique dans d'autres recherches (Seminowicz et al., 2013; Turner, Holtzman et Mancl, 2007). PROSPECT peut être modifié pour intégrer des stratégies telles que la restructuration cognitive (p. ex. déterminer et recadrer les pensées négatives automatiques au sujet des symptômes comme la douleur, l'anxiété et la dépression) (Beck et al., 2010) afin de cibler des variables cognitives telles que le catastrophisme dans les études subséquentes.

\section{IMPLICATIONS}

La nature de cette recherche a plusieurs répercussions pour les infirmières cliniciennes. Malgré les résultats négatifs de cette étude, les infirmières devraient continuer de préconiser l'utilisation de stratégies d'autogestion chez les personnes présentant des symptômes liés au traitement contre le cancer. Plus précisément, une étude intégrative de Hammer et collègues (2015) a examiné 46 articles mettant à l'essai des interventions d'autogestion chez les personnes atteintes de cancer (toutes des études dirigées par des chercheurs en sciences infirmières); cette étude a fait ressortir que plusieurs des interventions d'autogestion examinées avaient eu des effets positifs sur les symptômes liés au traitement du cancer (notamment douleur, fatigue, dépression et anxiété) (Hammer et al., 2015). De plus, les stratégies d'autogestion ne sont associées à aucun effet secondaire notable et peuvent être administrées en même temps que les traitements standards. Toutefois, avant de mettre en œuvre des stratégies d'autogestion, les infirmières doivent évaluer la volonté et la capacité de leurs patients à gérer eux-mêmes les symptômes. Par la suite, les infirmières doivent choisir des stratégies d'autogestion qui correspondent aux préférences et aux capacités de leurs patients ainsi qu'à leur horaire (rendez-vous, traitements, vie personnelle...) (McCorkle et al., 2011).

\section{LIMITES}

Cette étude comporte des limites. Létude n'était pas assez puissante, ce qui augmente la probabilité de trouver un résultat faussement négatif (erreur de type II). Les participants n'ont interagi avec le site Web PROSPECT que pendant huit semaines. Par conséquent, ils n'ont peut-être pas eu assez de temps pour apprendre et intégrer les stratégies du site Web dans leur vie quotidienne afin d'influencer les changements de comportement liés à la gestion de la douleur. De même, en raison de la nature autoguidée de l'intervention, les participants n'ont peut-être pas reçu la dose optimale de l'intervention afin de diminuer les symptômes liés à la douleur. Enfin, bien que nous ayons recueilli des données sur le temps que les participants ont passé à utiliser PROSPECT chaque semaine (Knoerl et al., 2017), nous n'avons pas recueilli de données sur 
la fréquence à laquelle les participants ont utilisé des modules spécifiques dans cet outil. Il est donc possible que les participants n'aient pas utilisé systématiquement les modules contenant des stratégies liées à l'anxiété, à la dépression, à la fatigue et/ou aux troubles du sommeil. Malgré ces limites, les résultats de cette étude contribuent à la documentation de plus en plus abondante sur l'identification des médiateurs de diminution de l'intensité de la douleur à la suite de la gestion cognitivocomportementale de la douleur afin de mieux comprendre comment ce traitement peut contribuer à améliorer la gestion de la douleur.

\section{CONCLUSION}

En conclusion, aucun des médiateurs hypothétiques de la douleur chronique de la NPCI n'était significatif. En raison de la petite taille de l'échantillon, il faudrait réévaluer l'effet médiateur des symptômes concomitants de la NPCI dans le cadre d'une étude de plus grande envergure. De plus, l'intervention avec PROSPECT devrait peut-être être modifiée à l'avenir pour amplifier son effet sur les cibles potentielles de médiation. En déterminant les médiateurs d'intensité de la

\section{RÉFÉRENCES}

Barsevick, A., Frost, M., Zwinderman, A., Hall, P., \& Halyard, M. (2010). I'm so tired: Biological and genetic mechanisms of cancerrelated fatigue. Quality of Life Research, 19(10), 1419-1427. http://doi. org/10.1007/s11136-010-9757-7

Bartlett, S.J., Orbai, A.-M., Duncan, T., DeLeon, E., Ruffing, V., CleggSmith, K., \& Bingham, C.O. (2015). Reliability and validity of selected PROMIS measures in people with rheumatoid arthritis. PLOS ONE, 10(9), e0138543. http://doi.org/10.1371/journal.pone.0138543

Beck, J.S. (2010). Cognitive therapy. In the Corsini Encyclopedia of Psychology. Hoboken, NJ, USA: John Wiley \& Sons, Inc. http:// doi.org/10.1002/9780470479216.corpsy0198

Beijers, A., Mols, F., Dercksen, W., Driessen, C., \& Vreugdenhil, G. (2014). Chemotherapy-induced peripheral neuropathy and impact on quality of life 6 months after treatment with chemotherapy. The Journal of Community and Supportive Oncology, 12(11), 401-6. http:// doi.org/10.12788/jcso.0086

Boakye, P.A., Olechowski, C., Rashiq, S., Verrier, M.J., Kerr, B., Witmans, M., ... Dick, B.D. (2016). A critical review of neurobiological factors involved in the interactions between chronic pain, depression, and sleep disruption. The Clinical Journal of Pain, 32(4), 327-36. http://doi.org/10.1097/ AJP.0000000000000260

Cella, D., Yount, S., Rothrock, N., Gershon, R., Cook, K., Reeve, B., ... PROMIS Cooperative Group. (2007). The Patient-Reported Outcomes Measurement Information System (PROMIS): Progress of an NIH roadmap cooperative group during its first two years. Medical Care, 45(5 Suppl. 1), S3-S11. http://doi.org/10.1097/01. mlr.0000258615.42478.55

Cleeland, C.S., \& Ryan, K.M. (1994). Pain assessment: global use of the Brief Pain Inventory. Annals of the Academy of Medicine, Singapore, 23(2), 129-38. Retrieved from http://www.ncbi.nlm.nih. gov/pubmed/8080219

DasMahapatra, P., Chiauzzi, E., Pujol, L.M., Los, C., \& Trudeau, K.J. (2015). Mediators and moderators of chronic pain outcomes in an online self-management program. The Clinical Journal of Pain, 31(5), 404-13. http://doi.org/10.1097/AJP.0000000000000125 douleur chez les personnes aux prises avec des douleurs liées à la NPCI, nous pourrons mieux cibler les stratégies comportementales au moyen de facteurs connus pour gérer l'intensité de la douleur.

\section{DIVULGATION}

Les auteurs déclarent n'avoir aucun conflit d'intérêts. La préparation de ces travaux a été rendue possible grâce à la bourse de nouveau chercheur de la University of Michigan School of Nursing et à la subvention de recherche Rackham pour étudiant à la maîtrise. La source de financement n'a joué aucun rôle dans la structure de létude, la collecte et l'analyse de données ou la rédaction de cet article. La création du site Web PROSPECT (PROactive Self-management Program for Effects of Cancer Treatment [programme d'autogestion proactive des effets du traitement contre le cancer]) était soutenue par la Damon Runyon Cancer Research Foundation (CI-53-10) accordé à Norah Lynn Henry. PROSPECT s'est inspiré du site Web Fibroguide.com qui a été conçu grâce aux subventions portant les numéros R01-AR050044 (NIAMS/NIH) et DAMD 17-00-2-0018 (Département de la Défense).

Hammer, M.J., Ercolano, E.A., Wright, F., Dickson, V.V., Chyun, D. \& Melkus, G.D. (2015). Self-management for adult patients with cancer. Cancer Nursing, 38(2), E10-E26. http://doi.org/10.1097/ NCC. 0000000000000122

Hershman, D.L., Lacchetti, C., Dworkin, R.H., Lavoie Smith, E.M. Bleeker, J., Cavaletti, G., ... Oncology, A.S. of C. (2014). Prevention and management of chemotherapy-induced peripheral neuropathy in survivors of adult cancers: American Society of Clinical Oncology clinical practice guideline. Journal of Clinical Oncology: Official Journal of the American Society of Clinical Oncology, 32(18), 1941-1967. http://doi.org/10.1200/JCO.2013.54.0914

Hershman, D.L., Till, C., Wright, J.D., Awad, D., Ramsey, S.D., Barlow, W.E., ... Unger, J. (2016). Comorbidities and risk of chemotherapyinduced peripheral neuropathy smong participants 65 years or older in Southwest Oncology Group Clinical Trials. Journal of Clinical Oncology, 34(25), 3014-3022. http://doi.org/10.1200/ JCO.2015.66.2346

Imai, K., Keele, L., \& Tingley, D. (2010). A general approach to causal mediation analysis. Psychological Methods, 15(4), 309-334. http:// doi.org/10.1037/a0020761

Imai, K., Keele, L., Tingley, D., \& Yamamoto, T. (2015). Causal mediation analysis using $R *$. Retrieved from http://imai.princeton. edu

Jensen, K.B., Kosek, E., Wicksell, R., Kemani, M., Olsson, G., Merle, J.V, ... Ingvar, M. (2012). Cognitive Behavioral Therapy increases pain-evoked activation of the prefrontal cortex in patients with fibromyalgia. Pain, 153(7), 1495-503. http://doi.org/10.1016/j. pain.2012.04.010

Kautio, A.-L., Haanpää, M., Kautiainen, H., Kalso, E., \& Saarto, T. (2011). Burden of chemotherapy-induced neuropathy-A crosssectional study. Supportive Care in Cancer, 19(12), 1991-1996. http:// doi.org/10.1007/s00520-010-1043-2

Knoerl, R., Lavoie Smith, E.M., \& Weisberg, J. (2015). Chronic pain and cognitive behavioral therapy: An integrative review. Western Journal of Nursing Research (journal article). http://doi. org/0193945915615869 [pii] 
Knoerl, R., Smith, E.M.L., Barton, D.L., Williams, D.A., Holden, J.E., Krauss, J.C., \& LaVasseur, B. (2017). Self-guided online cognitive behavioral strategies for chemotherapy-induced peripheral neuropathy: A multicenter, pilot, randomized, wait-list controlled trial. The Journal of Pain. http://doi.org/10.1016/j.jpain.2017.11.009

Kroenke, K., Yu, Z., Wu, J., Kean, J., \& Monahan, P.O. (2014). Operating characteristics of PROMIS four-item depression and anxiety scales in primary care patients with chronic pain. Pain Medicine, 15(11), 1892-901. http://doi.org/10.1111/pme.12537

Larun, L., Brurberg, K.G., Odgaard-Jensen, J., \& Price, J.R. (2016). Exercise therapy for chronic fatigue syndrome. In L. Larun (Ed.), Cochrane Database of Systematic Reviews. Chichester, UK: John Wiley \& Sons, Ltd. http://doi.org/10.1002/14651858.CD003200. pub6

Little, T.D., Jorgensen, T.D., Lang, K.M., \& Moore, E.W.G. (2014). On the joys of missing data. Journal of Pediatric Psychology, 39(2), 151-162. http://doi.org/10.1093/jpepsy/jst048

McCorkle, R., Ercolano, E., Lazenby, M., Schulman-Green, D., Schilling, L.S., Lorig, K., \& Wagner, E.H. (2011). Self-management: Enabling and empowering patients living with cancer as a chronic illness. CA: A Cancer Journal for Clinicians, 61(1), 50-62. http://doi. org/10.3322/caac. 20093

McCracken, L.M., Gross, R.T., \& Eccleston, C. (2002). Multimethod assessment of treatment process in chronic low back pain: Comparison of reported pain-related anxiety with directly measured physical capacity. Behaviour Research and Therapy, 40(5), 585-94. Retrieved from http://www.ncbi.nlm.nih.gov/ pubmed/12038650

Nudelman, K.N.H., McDonald, B.C., Wang, Y., Smith, D.J., West, J.D., O’Neill, D.P., ... Saykin, A.J. (2016). Cerebral perfusion and Gray Matter Changes associated with chemotherapy-induced peripheral neuropathy. Journal of Clinical Oncology: Official Journal of the American Society of Clinical Oncology, 34(7), 677-83. http://doi. org/10.1200/JCO.2015.62.1276

Price, J.R., Mitchell, E., Tidy, E., \& Hunot, V. (2008). Cognitive behaviour therapy for chronic fatigue syndrome in adults. In J.R. Price (Ed.), Cochrane Database of Systematic Reviews (p. CD001027). Chichester, UK: John Wiley \& Sons, Ltd. http://doi. org/10.1002/14651858.CD001027.pub2

R Development Core Team. (2017). R: A language and environment for statistical computing. Retrieved from https://www.r-project.org

Ritterband, L.M., Thorndike, F.P., Ingersoll, K.S., Lord, H.R., GonderFrederick, L., Frederick, C., ... Morin, C.M. (2017). Effect of a webbased cognitive behavior therapy for insomnia intervention with 1-year follow-up: A randomized clinical trial. JAMA Psychiatry, 74(1), 68-75. http://doi.org/10.1001/jamapsychiatry.2016.3249
Seminowicz, D.A., Shpaner, M., Keaser, M.L., Krauthamer, G.M., Mantegna, J., Dumas, J.A., ... Naylor, M.R. (2013). Cognitivebehavioral therapy increases prefrontal cortex gray matter in patients with chronic pain. The Journal of Pain: Official Journal of the American Pain Society, 14(12), 1573-84. http://doi.org/10.1016/j. jpain.2013.07.020

Smith, E.M.L., Bridges, C.M., Kanzawa, G., Knoerl, R., Kelly, J.P., Berezovsky, A., \& Woo, C. (2014). Cancer treatment-related neuropathic pain syndromes-Epidemiology and treatment: An update. Current Pain and Headache Reports, 18(11), 459. http://doi. org/10.1007/s11916-014-0459-7

Smith, E.M.L., Cohen, J.A., Pett, M.A., \& Beck, S.L. (2011). The validity of neuropathy and neuropathic pain measures in patients with cancer receiving taxanes and platinums. Oncology Nursing Forum, 38(2), 133-142.

Smith, E.M.L., Pang, H., Ye, C., Cirrincione, C., Fleishman, S., Paskett, E.D., ... Alliance for Clinical Trials in Oncology. (2015). Predictors of duloxetine response in patients with oxaliplatininduced painful chemotherapy-induced peripheral neuropathy (CIPN): A secondary analysis of randomised controlled trial CALGB/alliance 170601. European Journal of Cancer Care. http:// doi.org/10.1111/ecc.12421

Smith, E.M., Pang, H., Cirrincione, C., Fleishman, S., Paskett, E.D., Ahles, T., ... Oncology, A. for C. T. in. (2013). Effect of duloxetine on pain, function, and quality of life among patients with chemotherapy-induced painful peripheral neuropathy: A randomized clinical trial. JAMA, 309(13), 1359-1367. http://doi. org/10.1001/jama.2013.2813

Tingley, D., Yamamoto, H.T., Kentaro, M., Princeton, H., Keele, L., State, P., \& Imai, K. (2014). Mediation: R Package for Causal Mediation Analysis. Journal of Statistical Software.

Turner, J.A., Holtzman, S., \& Mancl, L. (2007). Mediators, moderators, and predictors of therapeutic change in cognitive-behavioral therapy for chronic pain. Pain, 127(3), 276-86. http://doi. org/10.1016/j.pain.2006.09.005

Yu, L., Buysse, D.J., Germain, A., Moul, D.E., Stover, A., Dodds, N.E., ... Pilkonis, P.A. (2012). Development of short forms from the PROMISTM Sleep Disturbance and Sleep-Related Impairment Item Banks. Behavioral Sleep Medicine, 10(1), 6-24. http://doi.org/10 $.1080 / 15402002.2012 .636266$

Zachariae, R., Lyby, M.S., Ritterband, L.M., \& O’Toole, M.S. (2016). Efficacy of internet-delivered cognitive-behavioral therapy for insomnia-A systematic review and meta-analysis of randomized controlled trials. Sleep Medicine Reviews, 30, 1-10. http://doi. org/10.1016/j.smrv.2015.10.004

Zhuo, M. (2016). Neural mechanisms underlying anxiety-Chronic pain interactions. Trends in Neurosciences, 39(3), 136-45. http://doi. org/10.1016/j.tins.2016.01.006 